\title{
BreastCore
}

\section{Romancing the Boost - the Higher, the Better?}

\author{
Felix Sedlmayer ${ }^{\mathrm{a}}$ Marie-Luise Sautter-Bihl ${ }^{\mathrm{b}} \quad$ Rolf Sauer $^{\mathrm{c}}$ \\ a Department of Radiotherapy, Paracelsus Medical University Hospital, Salzburg, Austria; \\ ${ }^{b}$ Department of Radiotherapy, Municipal Hospital, Karlsruhe, Germany; \\ ${ }^{\mathrm{c}}$ Department of Radiotherapy, University Hospital Erlangen, Germany
}

In terms of radiobiology, the probability of tumor control is essentially a function of dose, with a direct (sigmoid) correlation, predominantly irrespective of the cancer type. In practice, the ability to deliver ultimately tumoricidal doses is frequently limited by the tolerance of surrounding organs at risk for radiation damage, especially when microscopic spread involves critical amounts of radiosensitive tissues. Permanent local tumor control, however, is one of the prerequisites for definitive cure from cancer: the higher the rate of local control, the lower the risk of subsequent systemic spread with consecutive death from disease. As early as 1997, Sam Hellman was the first to summarize this thesis in an extremely concise phrase: to 'stop metastases at their source' [1].

In conservatively operated breast cancer, where local control was long believed to be rather irrelevant for the further course of disease, the opposite was repeatedly proven by the analyses of the Early Breast Cancer Trialists' Collaborative Group (EBCTCG): On average, in all patients, about 1 breast cancer death was avoided at 15 years for every 4 recurrences avoided by year 10. Little variation of the proportional benefit was seen in the different prognostic subgroups. In contrast, the absolute benefit from radiotherapy substantially depended on the patient characteristics in terms of prognostic factors $[2,3]$.

Considering the tumor bed as the most predetermined site for subclinical residual tumor, a local dose escalation has proven to lower in-breast recurrence rates most effectively. The high value of an additional 16-Gy booster dose to the tumor bed either by fractionated external electron beam treatment or by brachytherapy was corroborated in a follow-up analysis of the European Organisation for Research and Treatment of Cancer (EORTC) trial data, which prompted the present editorial [4].

Between 1989 and 1996, 2,657 patients were randomly assigned to receive no radiation boost and 2,661 patients were randomly assigned to receive a boost. The median follow-up was 17.2 years. Ipsilateral breast tumor recurrence was the first treatment failure for 354 patients (13\%) in the no-boost group versus 237 patients (9\%) in the boost group (hazard ratio (HR) 0.65 ; $99 \%$ confidence interval (CI) $0.52-0.81 ; \mathrm{p}<0.0001$ ). The 20 -year cumulative incidence of ipsilateral breast tumor recurrence was $16.4 \%$ in the no-boost group versus $12.0 \%$ in the boost cohort. Mastectomies as first salvage treatment for ipsilateral breast tumor recurrence were performed in 279 (79\%) of 354 patients in the no-boost group versus 178 (75\%) of 237 in the boost group. However, despite higher local control rates in all boost cohorts, and unlike the EBCTCG findings, the authors found no overall survival benefit at 20 years. At this point of time, survival was $59.7 \%$ in the boost group versus $61.1 \%$ in the no-boost group. The authors explained these contradictory results by a lower proportion of node-positive patients (about $20 \%$ ), in whom the survival benefit of radiotherapy was recorded most pronouncedly in the EBCTCG analyses. More importantly, they hypothesized that, in their trial, salvage mastectomy treatment for in-breast recurrences eliminated the local problem well enough before systemic spread could occur.

To our mind there are 2 additional aspects to be considered: If analysis endpoints are set back long enough, every in-between survival difference will likely disappear. Since the majority of patients were postmenopausal at the time of study entry, many of them would not profit in terms of an advantage to be still experienced 2 decades later.

Second, the absolute number of local recurrence events was surprisingly high in all boost groups: In a comparable case mix, a cumulative $12 \%$ in-breast relapse rate after 20 years would be far beyond nowadays' expectations. Especially in younger patients, with an almost 25\% local relapse rate (LRR) despite boost, a possible effect of superior local control on survival was far from being maxed out. This is also true for the group of patients older than 60 years, with an LRR of $11.1 \%$ despite local dose escalation.

As a matter of fact, more recent studies on the effect of dose escalation in high-risk patient selections (e.g. age below 40 years) do not yet have this longitudinal power of the EORTC trial. But at interim analyses at 5-7 years, even these younger patients do not

\section{KARGER}

Fax +497614520714 (c) 2015 S. Karger GmbH, Freiburg

$1661-3791 / 15 / 0104-0237 \$ 39.50 / 0$
Prim. Univ.-Prof. Dr. Felix Sedlmayer

Universitätsklinik für Radiotherapie und Radio-Onkologie

Landeskrankenhaus Salzburg, Universitätsklinikum der PMU

Müllner Hauptstrasse 48, 5020 Salzburg, Austria

F.Sedlmayer@salk.at 
show in-breast recurrence rates higher than $1.5 \%[5,6]$. In older low-risk patients, the LRR might nowadays be kept far below $1 \%$ following whole-breast irradiation (WBI) plus boost $[3,7,8]$. Throughout the last 2 decades, LRRs after breast-conserving treatment have continuously decreased, which is attributable to a variety of factors: broad evolvement of preoperative diagnostic means, higher quality in pathological work-up, individualization, and refinement of operative strategies, as well as additive local effects of modern systemic treatment. However, the highest contribution in controlling remnant in-breast disease still comes from radiotherapy. Throughout the last 10-15 years, reliable methods for identifying and thoroughly irradiating a tumor bed have evolved dramatically, including the introduction of intraoperative radiotherapy as extremely precise boost technique $[3,9]$.

Of note, when the EORTC boost trial was initiated, there were no established quality assurance (QA) guidelines for tumor bed localization, leaving a lot of uncertainty with regard to geographical misses in boost delivery. To our mind, this is the most likely explanation for the high failure rates in spite of putatively sufficient doses. In light of these facts, it does not seem logical to question the appropriateness of an additional boost for subgroups of patients before the full potential of dose effects has been tapped. This also applies to women over 60 years of age for whom proof of an overall survival advantage will remain ultimately challenging. Nonetheless, freedom from second therapy (and especially salvage mastectomy) per se is invaluable. Therefore, the conclusion of the authors of the EORTC boost trial [4] that 'the extra radiation dose can be avoided in most patients older than age 60 years' may be scrutinized. In contrast, we suggest offering a boost in addition to WBI to all patients who appear biologically and mentally fit enough not only to experience but also to appreciate the benefit of improved local control [3].

Thus, radiation oncologists should not only seek to better discriminate groups at different risks for local relapse and identify crucial features in tumor characteristics beyond age (such as triple-negative breast cancer (TNBC)), but they are also obliged to translate these findings into new dose concepts by various means, introducing an arsenal of growingly sophisticated radiation techniques to fully exploit both specific tumor biology and geometric precision.

\section{Disclosure Statement}

The authors have no conflicts of interest.

\section{References}

1 Hellman S: Stopping metastases at their source. N Engl J Med 1997;337:996-997.

- Early Breast Cancer Trialists' Collaborative Group (EBCTCG), Darby S, McGale P, Correa C, Cutter D, Duane F, Ewertz M, Gray R, Mannu G, Peto R, Whelan T, Wang Y, Wang Z, Darby S: Effect of radiotherapy after breast-conserving surgery on 10-year recurrence and 15-year breast cancer death: meta-analysis of individual patient data for 10,801 women in 17 randomised trials. Lancet 2011;378:1707-1716.

3 Sedlmayer F, Sautter-Bihl ML, Budach W, Dunst J, Fastner G, Feyer P, Fietkau R, Haase W, Harms W, Souchon R, Wenz F, Sauer R; Breast Cancer Expert Panel of the German Society of Radiation Oncology (DEGRO): DEGRO practical guidelines: radiotherapy of breast cancer I: radiotherapy following breast conserving therapy for invasive breast cancer. Strahlenther Onkol 2013;189:825-833.

4 Bartelink H, Maingon P, Poortmans P, Weltens C, Fourquet A, Jager J, Schinagl D, Oei B, Rodenhuis C, Horiot JC, Struikmans H, Van Limbergen E, Kirova Y, Elkhuizen P, Bongartz R, Miralbell R, Morgan D, Dubois JB, Remouchamps V, Mirimanoff RO, Collette S, Collette L; European Organisation for Research and Treatment of Cancer Radiation Oncology and Breast Cancer Groups: Whole-breast irradiation with or without a boost for patients treated with breast-conserving surgery for early breast cancer: 20-year follow-up of a randomised phase 3 trial. Lancet Oncol 2015;16:47-56.
Bartelink H, Bourgier C, Elkhuizen P: Has partial breast irradiation by IORT or brachytherapy been prematurely introduced into the clinic? Radiother Oncol 2012;104:139-142.

6 Fastner G, Sedlmayer F, Merz F, Deutschmann H, Reitsamer R, Menzel C, Stierle C, Farmini A, Fischer T, Ciabattoni A, Mirri A, Hager E, Reinartz G, Lemanski C, Orecchia R, Valentini V: IORT with electrons as boost strategy during breast conserving therapy in limited stage breast cancer: long term results of an ISIORT pooled analysis. Radiother Oncol 2013;108:279-286.

7 Veronesi U, Orecchia R, Maisonneuve P, Viale G, Rotmensz N, Sangalli C, Luini A, Veronesi P, Galimberti V, Zurrida S, Leonardi MC, Lazzari R, Cattani F, Gentilini O, Intra M, Caldarella P, Ballardini B: Intraoperative radiotherapy versus external radiotherapy for early breast cancer (ELIOT): a randomised controlled equivalence trial. Lancet Oncol 2013;14:1269-1277.

8 Pötter R, Gnant M, Kwasny W, Tausch C, HandlZeller L, Pakisch B, Taucher S, Hammer J, LuschinEbengreuth G, Schmid M, Sedlmayer F, Stierer M, Reiner G, Kapp K, Hofbauer F, Rottenfusser A, Pöstlberger S, Haider K, Draxler W, Jakesz R; Austrian Breast and Colorectal Cancer Study Group: Lumpectomy plus tamoxifen or anastrozole with or without whole breast irradiation in women with favorable early breast cancer. Int J Radiat Oncol Biol Phys 2007;68: 334-340.
Krengli M, Calvo FA, Sedlmayer F, Sole CV, Fastner G, Alessandro M, Maluta S, Corvò R, Sperk E, Litoborski M, Pisani C, Fillini C, Fusconi F, Osti MF, Tomio L, Marsiglia H, Ciabattoni A, Polkowski W, Di Grazia A, Gava A, Kuten A, Iotti C, Gonzalez C, Sallabanda M, Dubois JB, Catalano G, Valentini V: Clinical and technical characteristics of intraoperative radiotherapy. Analysis of the ISIORT-Europe database. Strahlenther Onkol 2013;189:729-737.

10 Liedtke C, Thill M, Hanf V, Schütz F: AGO recommendations for the diagnosis and treatment of patients with early breast cancer: update 2014. Breast Care 2014;9:189-200. 